\title{
Resilience and associated factors in women survivors of Intimate Partner Violence:
}

\section{a systematic review}

\author{
Natalia Fernández-Álvarez*, Yolanda Fontanil, and Ángeles Alcedo
}

Universidad de Oviedo (Spain)

\begin{abstract}
Título: Resiliencia y factores asociados en mujeres supervivientes de Violencia de Género en la Pareja: una revisión sistemática.

Resumen: Gran parte de los estudios sobre Violencia de Género en la Pareja (VGP) presentan a las mujeres como víctimas pasivas, pero en los últimos años el número de investigaciones centradas en fortalezas está aumentando. El objetivo del presente estudio es revisar la literatura empírica sobre resiliencia en mujeres supervivientes de VGP para: (a) analizar el consenso en la definición y evaluación de resiliencia; (b) estudiar los correlatos del constructo; y (c) reflexionar sobre la aplicabilidad de este conocimiento en las políticas y la práctica profesional. Siguiendo las guías PRIS MA, se realizó una revisión sistemática en las bases de datos Scopus, WoS y PsycINFO, encontrando 42 artículos publicados en inglés o español sin restricción temporal. Los resultados muestran que los estudios adoptan definiciones de resiliencia tanto orientadas al proceso como al rasgo y a los resultados, y que el método deevaluación difiere entre investigaciones. Entre los correlatos de resiliencia se encontraron factores individuales, relacionales y contextuales. Se necesita un consenso teórico y profundizar en los mecanismos por los cuales los factores de vulnerabilidad o protección afectan a grupos con riesgos específicos. Por último, los gobiernos e instituciones deberían emprender acciones de prevención y de apoyo a las mujeres e hijos/as.
\end{abstract}

Palabras clave: Violencia de Género en la Pareja. Violencia de Género. Resiliencia. Fortalezas. Políticas. Revisión sistemática.
Abstract: Most research on Intimate Partner Violence against women (IPV) has tended to present women as passive victims, but in recent years, there has been an increase in studies adopting a strengths-based approach The aim of the present study is to review the empirical literature about resilience in women survivors of IPV to (a) analyze the consensus on the definition andassessment of resilience; (b) study the correlates of resilience; and (c) reflect on the applicability of such knowledge in policies and professional practice. Following PRISMA guidelines, a systematic search was carried out in the Scopus, WoS and PsycINFO databases. The search yielded 42 articles published in English or Spanish over an unlimited timeframe. Results showed that process-oriented, trait-oriented, and outcomesoriented definitions of resilience were all adopted in the research and that assessment methods also differed across studies. Individual, relational, and contextual factors were found as resilience correlates.

There is a need for a theoretical consensus and for research on the mechanisms through which vulnerability or protective factors exert their influence on groups with specific risk conditions. Finally, governments and institutions should take actions to support women and children and to prevent future IPV.

Keywords: Intimate Partner Violence. Gender Violence. Resilience. Strengths. Policies. Systematic review.

\section{Introduction}

Intimate Partner Violence against women (IPV; also known as Gender Violence in the Spanish legal framework) is a public health problem with severe consequences for a high proportion of women around the world (Delegación del Gobierno contra la Violencia de Género [DGVG], 2019; World Health Organization [WHO], 2013). Much research in this field has focused on the damage that violence causes in different life areas, conceptualizing women as passive victims of their circumstances (Arias et al., 2016). Offering a less partial and pathologizing view of women, Gondolf and Fisher (1988) proposed the survivor theory. Their approach recognized that mistreated women are active agents using diverse and innovative coping strategies to guarantee their own safety and to protect their children. In fact, studies have found that women usually seek formal and/or informal support, despite the obstacles of fear, guilt, physical and emotional discomfort, economic constraints, and absence or inadequacy of community resources (Fontanil et al., 2020; Lelaurain et al., 2017).

As a result of Gondolf and Fisher's contributions, a research approach focusing on women's strengths emerged.
From this perspective, it is assumed that women employ various resources to cope with and overcome the violence. These resources must be promoted since trauma and adversity are change opportunities. Studies have found that women are able to survive and draw positive learnings from their experience, with many of them subsequently becoming involved in activism work and actions to help other women who suffer or have suffered IPV (Crann \& Barata, 2016; Fernández-Sánchez \& López-Zafra, 2019). After decades of research, it is important to reflect on the extent to which existing knowledge can guide future policies and professional practices. However, as Anderson et al. (2012) point out, even though suffering should not be taken as the central component of the women's identity, nor should the severity of their experience be minimized. The trauma recovery process is characterized by the co-occurrence of positive and negative experiences, fluctuating between states of suffering and personal growth, or resilience and psychopathology; denial of any of these events implies ignoring a part of the women's reality (Fontanil et al., 2020; Humphreys, 2003; Rodríguez et al., 2008).

From this perspective, based on the strengths of women to overcome the violent relationship, the pivotal concept of resilience emerges. 


\section{Resilience: still unclear}

The construct of resilience has become a core element in the analysis of vulnerable populations from a strengths-based approach. Even so, there is still a lack of consensus about how it should be defined and assessed (Bushati, 2020; Chmitorz et al., 2018; Stainton et al., 2018; Ungar, 2019).

In relation to the resilience concept, trait-oriented, outcomes-oriented, and process- oriented definitions coexist in resilience literature. Trait-oriented definitions describe resilience as a personality trait that is an internal, relatively stable, and consistent personal characteristic (Hu et al., 2015). Outcomesoriented definitions consider that resilience is the ability to maintain a state of equilibrium; the main resilience indicator is the result, consequence, or impact that adversity has on the person's circumstances (Bonanno, 2004). Finally, process-oriented definitions support the dynamic character of resilience, which operates through a multitude of individual and contextual resources in constant interaction with each other. From this perspective, resilience is characterized by temporal and contextual specificities, that is, the same person can show resilience in certain environments or domains but not in others, or can be resilient at a specific life stage but not in all (Bushati, 2020; Stainton et al., 2018; Ungar, 2019; Ungar \& Theron, 2019). Other investigations support an ecological view of resilience, similar to process-oriented definitions (Fontanil \& Alcedo, 2018; Fontanil et al., 2020).

Various methods have been used to assess resilience. Some studies have measured resilience by the presence/absence of psychopathology, while others have explored related protective factors (for example, self-efficacy) or used specific resilience scales (Stainton et al., 2018; Ungar \& Theron, 2019).

In response to the lack of consensus, Ungar (2008) proposed a socio-ecological model in which resilience is considered as both the capacity to individually and collectively negotiate and access the psychological, social, cultural, and physical resources that sustain well-being, and the ability to experience them in culturally meaningful ways. Resilience is therefore composed of multiple and interrelated dimensions and factors present at different ecological levels. These resilience factors include relationships, defined identity, power/control, social justice, access to material resources, sense of cohesion/belonging, and cultural adherence (Ungar, 2013).

Contextual and dynamic definitions of resilience have the advantage of recognizing the heterogeneity of functioning shown by people who overcome adverse situations and, in the specific case of IPV against women, remove the focus from the negative discourse surrounding women exposed to IPV (Ahmad et al., 2013; Howell et al., 2018). At the same time, ecological definitions highlight those resilience factors that are potentially variable over time versus those that are more stable (Howell et al., 2018; Kuijpers et al., 2012). Process-oriented definitions are useful for analyzing women's resilience in the different phases of the violent relationship and after the break up, detecting which are the main resilience correlates at each stage (Labronici,
2012). Also, from an ecological perspective, political efforts to provide women with resources to cope with their circumstances are vital (Fontanil \& Alcedo, 2018; Fontanil et al., 2020).

\section{Correlates of resilience}

Despite these conceptual and methodological issues, research on resilience in recent decades has identified a series of consensual and cross-cutting resilience factors that are present at the individual, relational, and contextual ecological levels. In the individual sphere, a range of resilience-enhancing variables have been reported, including problem-solving skills, agency and self-efficacy, sense of humor, adaptability, and meaning making. In the relational domain, the literature describes a variety of characteristics related to the quality of relationships, for example, security, trust, nurture, care, stability, and acceptance. Finally, the contextual resilience resources include sense of belonging, educational and employment resources, service provision, and policies (Bushati, 2020; Liebenberg et al., 2017; Stainton et al., 2018; Ungar \& Theron, 2019).

Until recently, women survivors of IPV tended to be a largely disregarded collective in research on resilience. Over the last number of years however, the study of this construct in the field of IPV has attracted increased research attention (Fernández-Sánchez \& López- Zafra, 2019; Howell et al., 2018; LópezFuentes \& Calvete, 2015), resulting in the identification of different factors related to resilience in this specific population. In this context, there is a clear need to compile the findings obtained in this field to determine if there is sufficient empirical evidence to enhance resilience in women survivors of IPV and to suggest future research directions.

With this objective, the present study reviews the empirical literature on resilience in women survivors of IPV to (a) analyze the consensus on the definition and assessment of resilience; (b) study the correlates of resilience in women survivors of IPV; and (c) reflect on the applicability of such knowledge in policies and professional practice.

\section{Method}

A systematic review was carried out in accordance with PRISMA guidelines (Preferred Reporting Items for Systematic Reviews and Meta-Analyses; Moher et al., 2009) and the recommendations for the reporting of systematic reviews (RubioAparicio et al., 2018).

\section{Inclusion criteria}

The inclusion criteria did not apply any limits to publication date, context, and methodology or experimental design. Exclusion criteria were defined for document type, language, and the publication adaptation to the aims of the systematic review. A full list of the inclusion and exclusion criteria for the identification of relevant literature is shown in Table 1. 
Table 1. Inclusion and exclusion criteria.

\begin{tabular}{ll}
\hline Studies included (were) & Studies excluded (were) \\
\hline Empirical & Book chapters \\
& Ph.D. theses/dissertations Editorials \\
& Congress abstracts \\
& Theoretical and meta-analytic studies \\
Conducted with children & Conducted with men \\
& Conducted with clinicians (exclusively based on their reports without women reports) \\
Published in English or Spanish & Not published in English or Spanish \\
Published in any year & \\
Conducted in any setting & Studies that did not assess resilience \\
Studies that assessed resilience &
\end{tabular}

\section{Search strategy}

Literature searches were performed in PsycINFO, Scopus and all the Web of Science (WoS) bibliographic databases. Figure 1 presents the combinations of keywords entered intoWoS and a summary of the study selection process. Searches were repeated in all of the databases using the cor- responding keywords in the Spanish language. An additional four relevant articles were found through a manual search in Google Scholar, and another two by scanning references from the selected articles. Both hand-searching (Vassar et al., 2016) and reference list scanning (Liberati et al., 2009) are supplemental approaches that increase the quality of systematic reviews.

Figure 1

Summary of study selection process.

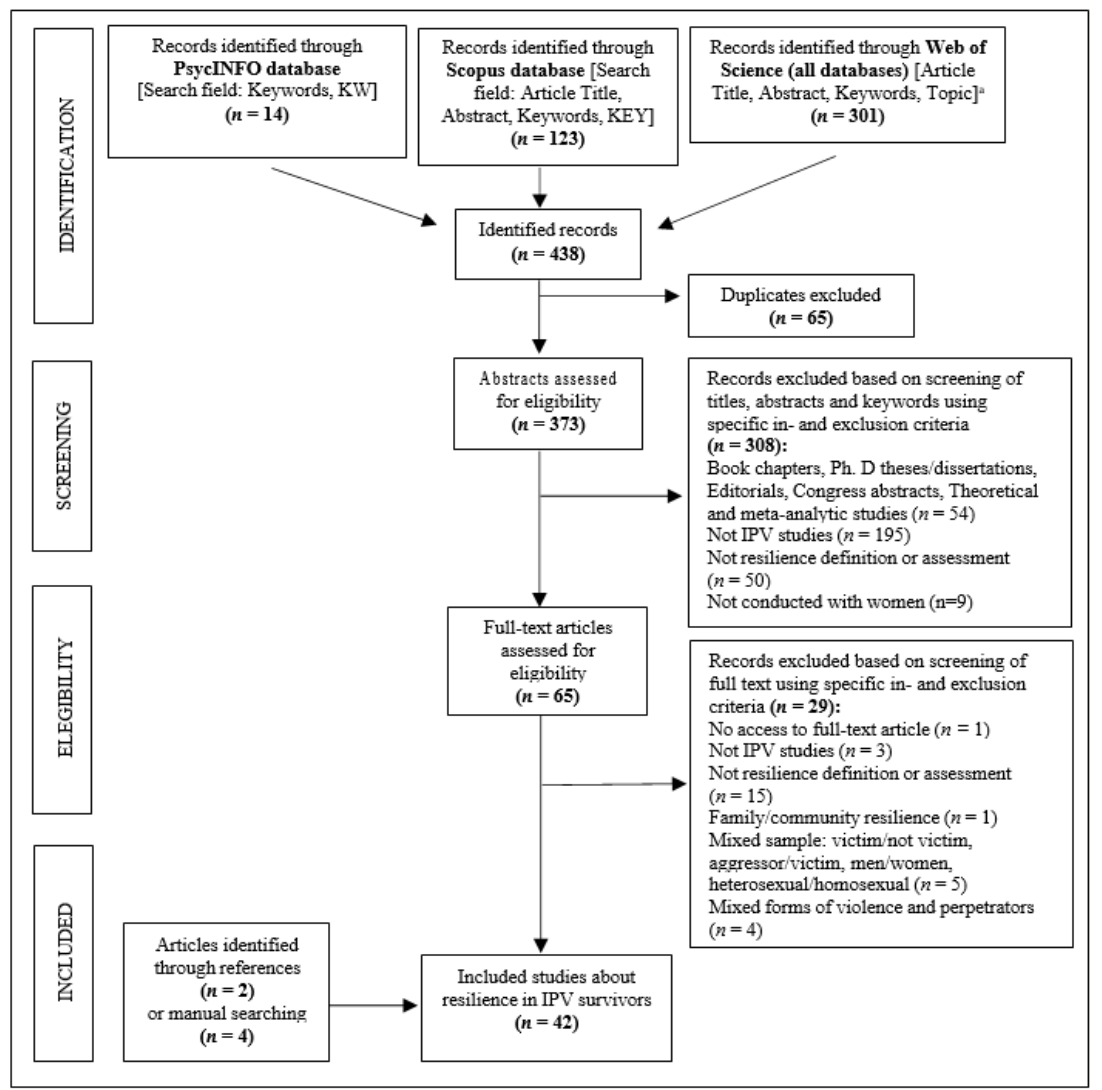

${ }^{a}$ The search keywords used in WoS: TOPIC: ("Intimate partner violence" OR "IPV" OR "Intimate partner aggression" OR "Partner violence " OR "Partner abuse" OR "Partner aggression" OR "Domestic aggression" OR "Domestic violence" OR "Domestic abuse" OR "Gender violence" OR "Domestic aggression" OR "Spouse abuse" OR "Spouse violence" OR "Spouse aggression" OR "Spousal abuse" OR "Spousal violence" OR "Spousal aggression" OR "Violence against women" OR "VAW" OR "battered woman" OR "mistreat*" OR "dating violence") AND TOPIC: ("Resilience" OR "Resilient" OR "Resiliency") AND TOPIC: ("Woman" OR "Women" OR "Female" OR "Females" OR "Girl" OR "Girls") NOT TOPIC: ("homosexual" OR "gay" OR "lesbian" OR "LGBTQI" OR "LGBTQI" OR "transgender" OR "transsexual" OR "same-sex relationship" OR "same sex relationship" OR "same-sex partner" OR "same sex partner"). Refined by: Type of document: (article), Language: (English OR Spanish). 
The search yielded 42 articles from 2000 to 2019. Articles included in the review are marked with an asterisk in the references section. To extract the data, a form was developed containing sociodemographic, methodological, and theoretical information. Results obtained were organized according to the following areas: (a) the aims of the systematic review (to analyze the consensus on the definition and assessment of resilience/to study the correlates of resilience in women survivors of IPV); and (b) the design of the selected studies (qualitative/quantitative).

\section{Results}

\section{Description of the studies}

Most of the reviewed studies were cross-sectional $(85.8 \%)$ and quantitative $(57.1 \%)$.

Among the studies that recruited sample from services $(81 \%)$ or from services and other sources $(7.1 \%)$, a high proportion recruited either from specific women's and IPV services $(46 \%)$ or from unspecific services (40.5\%); only $13.5 \%$ recruited sample from both types of services.

\section{Resilience definition and assessment}

Most of the studies, whether qualitative or quantitative/mixed-method, included at least one definition of resilience, as shown in Table 2. Qualitative inquiries presented process-oriented definitions more frequently than quantitative and mixed-method studies, which often defined resilience from a trait-oriented perspective. The outcomesoriented approach was the least common in both types of research.

Table 2

Approaches to defining resilience $(n=37)$.

\begin{tabular}{lll}
\hline & $n$ & $\%$ \\
\hline Qualitative studies providing a definition $(n=15)$ & & \\
\hline Process-oriented & 9 & 60 \\
Trait-oriented & 3 & 20 \\
Outcomes-oriented & 2 & 13.3 \\
Mixed-approach & 1 & 6.7
\end{tabular}

Quantitative and mixed-method studies providing a definition $(n=$ 22)

\begin{tabular}{lll}
\hline Process-oriented & 5 & 22.7 \\
Trait-oriented & 9 & 41 \\
Outcomes-oriented & 2 & 9 \\
Mixed-approach & 6 & 27.3 \\
\hline
\end{tabular}

Turning to the assessment approach (Table 3), a large number of qualitative studies used semi-structured interviews and frequently explored contextual factors and resilience changes over time. Quantitative articles usually employed a resilience scale. When Resilience was measured in this way, only The Resilience Research Centre Adult Resilience Measure (RRCARM; Liebenberg et al., 2012) comprehensively explored resilience from an ecological perspective, incorporating social/community inclusion, attachment and family support, and national and cultural identity as part of the assessment (Scrafford et al., 2019). Among the small number of quantitative studies that assessed resilience as an outcome, scores in depression and/or PTSD scales were the most frequent outcome variables (Machisa et al., 2018; Kramer et al., 2015), but studies also measured revictimization by a new partner (Herrero et al., 2018) and quality of life (Kuijpers et al., 2012). Only a few studies assessed resilience through a set of variables; in this case, contextual variables such as social support or marginality were also reported, but not from a dynamic perspective (Alvi et al., 2008; Cesario et al., 2014; McFarlane et al., 2014).

Table 3

Approaches to assessing resilience.

\begin{tabular}{lcc}
\hline & $n \%$ \\
\hline Qualitative studies $(n=17)$ & & \\
\hline SI & 10 & 58.8 \\
OI & 2 & 11.8 \\
FG & 2 & 11.8 \\
Multimethod (combining SI, OI, FG, O or LH) & 3 & 17.6 \\
\hline Quantitative $(n=25)$ & \\
\hline Resilience scale & 1872 \\
Set of variables & 3 & 12 \\
Resilience outcomes & 4 & 16 \\
\hline Note. SI: Semi-Structured Interview; OI: Open Interview; FG: Focus Group; &
\end{tabular}

\section{Correlates of resilience in women survivors of IPV}

The present systematic review found several factors associated with resilience at the individual, relational, and contextual ecological levels (Table 4).

The individual variables identified include personal characteristics such as problem-focused coping, sense of control, self-efficacy, self-esteem, sense of humor, optimism/hope, proactivity, adaptability, creativity, and spirituality. Studies also explored resilience processes, among these, identity reconstruction, reframing the violent relationship, setting goals, and personal growth.

One of the reviewed articles established physical activity as an enabler of women's resilience (López-Fuentes \& Calvete, 2015). The existing data suggest that resilience is positively correlated with perceived physical health (Humphreys, 2003) and negatively correlated with early labor and delivery (Scrafford et al., 2019), and medication consumption (Fernández-Sánchez \& López-Zafra, 2019). In contrast, FordGilboe et al. (2009) found that resilience had a direct effect on mental health but not on physical health.

As regards the correlates of resilience and psychological health, research has shown that higher resilience is positively correlated with psychological health and negatively correlated with anxiety-depression and post-traumatic stress symptoms. Choi et al. (2019) noted that resilience did not predict generalized anxiety scores when controlling for variability in the heterogeneity of women's past victimization. The au- 
thors posited that this was because economic and employment stability, social support, and the history of abuse had a stronger effect than resilience on the anxiety levels of their sample given the participants' characteristics (young women with children).

As for the relational factors, the ability of women to rebuild and expand their social network was key. Help-seeking and the use of support systems, both formal and informal, were positively correlated with resilience, as they were a way to access instrumental/material, informational, and emotional resources, while providing validation and models of strong and empowered women.

Many of the studies found that having children enhanced resilience. One article, however, suggested the opposite, that is, women without children had higher resilience scores and suffered less violence (Jaramillo-Vélez et al., 2005).

Some of the articles examined the influence of a history of child abuse and adversity on resilience. Herrero et al.
(2018) found that exposure to abuse during childhood increased the likelihood of a woman being non-resilient (more likely to have had more than one abusive partner). Other studies observed higher resilience scores in women who had been victimized during their childhood (Roditti et al., 2010; Schultz et al., 2009; Scrafford et al., 2019), but only Scrafford et al. (2019) reported statistically significant results.

Finally, among the contextual elements, the most frequently studied factor was formal support. As stated above, this systematic review acknowledged that formal support is positively related to resilience, as it is a way for women to access the resources they need in their day-to-day lives and generates a sense of community belonging.

Figure 2 contains a summary of the main contextual, relational, and individual factors associated with resilience in women survivors of IPV.

Table 4

Factors associated with resilience at the individual, relational, and contextual ecological levels

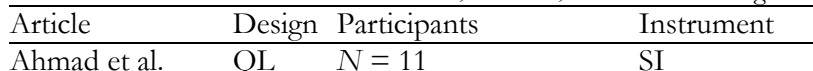

Factors associated with resilience

(2013)

Individual: willpower, problem-solving skills, self-efficacy, autonomy,optimism/hope, faith, goals, desire to stop transgenerational abuse.

Relational: seeking children's well-being, informal support, altruistic and activist actions.

Contextual: work/volunteering/hobbies/useful activities, formal support, sense of belonging.

\begin{tabular}{lllll}
\hline Crann \& Barata & QL & $N=16$ & SI & Individual: violence acknowledgment, resistance to cultur-
\end{tabular}

(2016) al norms and abuse sequelae, reframing the violent relationship, identity reconstruction, sense of control, self-esteem, optimism/hope, adaptability, personal growth.

Relational: new positive relationships, altruistic and activist actions.

Contextual: police contact.

\begin{tabular}{lllll}
\hline $\begin{array}{l}\text { Crawford et al. } \\
\text { (2009) }\end{array}$ & QL & $N=8$ & SI & $\begin{array}{l}\text { Individual: identity reconstruction, not recognizing own } \\
\text { resilience and agency*, external locus of control*, denial* }\end{array}$
\end{tabular}
(2009) minimization*, self- blaming*.

Relational: help-seeking for children, relationships with women survivors of IPV, unsupportive informal network*, altruistic and activist actions.

Contextual: employment, education, unsupportive community*, stigmatization*, gender roles and attitudes favorable to IPV*.

\begin{tabular}{llll}
\hline Davis (2002) & QL $\quad N=17$ & SI & Individual: sense of humor, optimism/hope, spirituality,
\end{tabular} control-free moments.

Contextual: informational resources, counseling, group therapy, formal support.

\begin{tabular}{|c|c|c|c|}
\hline $\begin{array}{l}\text { Drumm et al. } \\
\text { (2014) }\end{array}$ & QL $\quad N=42$ & SI & Individual: spirituality. \\
\hline $\begin{array}{l}\text { Fernández- } \\
\text { Sánchez \& } \\
\text { López-Zafra } \\
(2019) \\
\end{array}$ & $N=60$ & SI & $\begin{array}{l}\text { Individual: internal locus of control, hardiness, adaptabil } \\
\text { ity,psychological diagnostic*, medication consumption*. }\end{array}$ \\
\hline $\begin{array}{l}\text { Fogarty et al. } \\
\text { (2019) }\end{array}$ & $N=9$ & SI & $\begin{array}{l}\text { Individual: reframing the violent relationship. } \\
\text { Relational: seeking children's well-being, help-seeking. } \\
\text { Contextual: employment. }\end{array}$ \\
\hline
\end{tabular}




\begin{tabular}{|c|c|c|c|c|}
\hline Article & Design & Participants & Instrument & Factors associated with resilience \\
\hline $\begin{array}{l}\text { Gopal \& Nunlall } \\
\text { (2017) }\end{array}$ & QL & $\begin{array}{l}N=7 \\
\text { Clients }(n=4) \\
\text { Social workers }(n=3)\end{array}$ & $\begin{array}{l}\text { SI } \\
\text { FG }\end{array}$ & $\begin{array}{l}\text { Individual: violence acknowledgment, reframing the vio- } \\
\text { lent relationship, willpower, sense of control, opti- } \\
\text { mism/hope, proactivity. }\end{array}$ \\
\hline
\end{tabular}

Relational: seeking children's well-being, help-seeking, informal support.

Contextual: counseling, group programs for women survivors of IPV, formal support, supportive community.

\begin{tabular}{llll}
\hline Labronici (2012) QL $\quad N=5$ & OI & Individual: reframing the violent relationship, sense of
\end{tabular}

Relational: informal support.

Contextual: formal support.

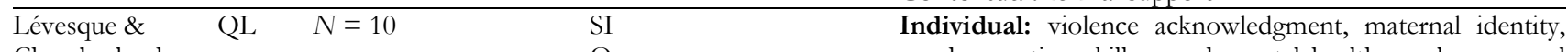

Chamberland $\quad \mathrm{O} \quad$ good parenting skills, good mental health, goals, personal (2016) growth.

Relational: seeking children's well-being, mother-child relationship, informal support.

Contextual: limited access to financial and material resources*, difficulties reconciling work, school, and childcare*.

\begin{tabular}{|c|c|c|}
\hline $\begin{array}{l}\text { López-Fuentes \& QL } \\
\text { Calvete (2015) }\end{array}$ & $\begin{array}{l}N=22 \quad \text { SI } \\
\text { Unrecovered }(n=10): \\
\text { presence of depression } \\
\text { or PTSD } \\
\text { Recovered }(n=12): \\
\text { absence of depression or } \\
\text { PTSD }\end{array}$ & $\begin{array}{l}\text { Individual: introspection, identity reconstruction, sense of } \\
\text { control, sense of humor, optimism/hope, creativity, altru- } \\
\text { ism, spirituality, focus on the present, physical activity, de- } \\
\text { pression and/or PTSD symptoms*,goals. } \\
\text { Relational: informal support. } \\
\text { Contextual: housing, formal support. }\end{array}$ \\
\hline $\begin{array}{l}\text { Schaefer et al. } \quad \text { QL } \\
(2019)\end{array}$ & $\begin{array}{l}N=56 \quad \text { FG } \\
\text { Mothers }(n=10) \\
\text { Service providers }(n= \\
46)\end{array}$ & $\begin{array}{l}\text { Individual: perseverance. } \\
\text { Relational: mother-child relationship, social network. }\end{array}$ \\
\hline $\begin{array}{l}\text { Shanthakumari et QL } \\
\text { al. (2014) }\end{array}$ & $N=16$ & $\begin{array}{l}\text { Individual: internal locus of control, active coping, self- } \\
\text { efficacy, self-esteem, optimism/hope, proactivity, persever- } \\
\text { ance, courage, dignity, altruism, faith. } \\
\text { Relational: seeking children's well-being, strength models } \\
\text { of other IPV survivors, altruistic and activist actions, infor- } \\
\text { mal support. } \\
\text { Contextual: employment. }\end{array}$ \\
\hline Taylor (2004) & $N=21$ & $\begin{array}{l}\text { Individual: reframing the violent relationship, identity re- } \\
\text { construction, optimism/hope, adaptability, creativity, } \\
\text { strength, spirituality, goals. } \\
\text { Relational: help-seeking, informal support, new positive } \\
\text { relationships, altruistic and activist actions. } \\
\text { Contextual: education, employment. }\end{array}$ \\
\hline $\begin{array}{l}\text { Trigueiro et al. } \quad \text { QL } \\
\text { (2014) }\end{array}$ & $N=8$ & $\begin{array}{l}\text { Individual: goals. } \\
\text { Relational: seeking children's well-being, strength models } \\
\text { of other IPVsurvivors. } \\
\text { Contextual: safe environment. }\end{array}$ \\
\hline $\begin{array}{l}\text { Werner-Wilson et QL } \\
\text { al. (2000) }\end{array}$ & $N=$ not provided & $\begin{array}{l}\text { Individual: active coping, proactivity, strength. } \\
\text { Relational: help-seeking, informal support. } \\
\text { Contextual: formal support. }\end{array}$ \\
\hline $\begin{array}{l}\text { Zalapa et al. } \\
(2012)\end{array}$ & $\begin{array}{l}\text { SI } \\
\mathrm{O} \\
\mathrm{LH}\end{array}$ & $\begin{array}{l}\text { Individual: problem-focused coping, agency, self-esteem, } \\
\text { creativity, perseverance, spirituality. } \\
\text { Relational: mother-child relationship, informal support. } \\
\text { Contextual: information, employment, self-help group, } \\
\text { psychological support. }\end{array}$ \\
\hline
\end{tabular}




\begin{tabular}{|c|c|c|c|c|}
\hline Article & Design & Participants & Instrument & Factors associated with resilience \\
\hline Alvi et al. (2008) & QT & $\begin{array}{l}N=117 \\
\text { Black }(n=77) \\
\text { Hmong }(n=40)\end{array}$ & $\begin{array}{l}\text { Massachusetts MothersI } \\
\text { Survey (Colton, 2001; } \\
\text { Raphael \& Tolman, 1997). C } \\
\text { "Overall, how would you } \\
\text { rate your physical health?" } \\
\text { Ad-hoc institutional support } \\
\text { scale. } \\
\text { Ad-hoc informal support } \\
\text { scale. }\end{array}$ & $\begin{array}{l}\text { Individual: race. } \\
\text { Relational: informal support. } \\
\text { Contextual: formal support. }\end{array}$ \\
\hline $\begin{array}{l}\text { Anderson et al. } \\
\text { (2012) }\end{array}$ & $\begin{array}{l}\text { Mixed- } \\
\text { method }\end{array}$ & $N=37$ & $\begin{array}{l}\text { SI } \\
\text { CD-RISC }\end{array}$ & $\begin{array}{l}\text { Individual: spirituality, PTSD symptoms. } \\
\text { Relational: informal support. } \\
\text { Contextual: formal support. }\end{array}$ \\
\hline $\begin{array}{l}\text { Canaval et al. } \\
\text { (2007) }\end{array}$ & QT & $N=100$ & RS & Individual: spirituality. \\
\hline $\begin{array}{l}\text { Canaval et al. } \\
\text { (2009) }\end{array}$ & QT & $\begin{array}{l}N=100 \\
(97 \text { for some analyses } \\
\text { because of missing data) }\end{array}$ & RS & $\begin{array}{l}\text { Individual: PTSD*, psychological distress*, obsessive- } \\
\text { compulsive symptoms*, interpersonal sensibility*, depres- } \\
\text { sion*, hostility*, psychoticism*. }\end{array}$ \\
\hline $\begin{array}{l}\text { Cesario et al. } \\
\text { (2014) }\end{array}$ & QT & $\begin{array}{l}N=106 \\
\text { Women who stayed } \leq \\
21 \text { days in shel- } \\
\text { ter/Women who stayed } \\
>21 \text { days in shelter } \\
\text { Women who received a } \\
\text { protection or-1 } \\
\text { der/Womenwho did not } \\
\text { receive a protection or- } \\
\text { der }\end{array}$ & $\begin{array}{l}\text { Safety Behavior Checklist } \\
\text { (McFarlane \& Parker, } \\
\text { 1994). } \\
\text { General Self-Efficacy Scale } \\
\text { (Schwarzer \& Jerusalem, } \\
\text { 1995). Norbeck Social Sup- } \\
\text {-port (Norbeck et al., 1981, } \\
\text { t1983). } \\
\text {-Koci Marginality Index } \\
\text { (KMI;Koci, 2004). }\end{array}$ & Contextual: access to shelter, access to justice service. \\
\hline Choi et al. (2018) & QT & $\begin{array}{l}N=80 \\
\text { VS group }(n=32) \text { : re- } \\
\text { ceived Volunteer Sup- } \\
\text { port (VS) } \\
\text { Non-VS group }(n=48) \text { : } \\
\text { did not receive VS }\end{array}$ & DRS 15 & Contextual: volunteer support. \\
\hline Choi et al. (2019) & QT & $N=79$ & DRS 15 & Individual: youth, motherhood. \\
\hline $\begin{array}{l}\text { de la Rosa et al. } \\
\text { (2015) }\end{array}$ & QT & $N=54$ & RS & $\begin{array}{l}\text { Individual: spirituality. } \\
\text { Relational: marital status, years of abuse. } \\
\text { Contextual: education, income. }\end{array}$ \\
\hline $\begin{array}{l}\text { Ford-Gilboe et al. } \\
(2009)\end{array}$ & QT & $N=309$ & RS & $\begin{array}{l}\text { Individual: global mental health, PTSD, depression. } \\
\text { Relational: severity of abuse, informal support. } \\
\text { Contextual: economic resources. }\end{array}$ \\
\hline $\begin{array}{l}\text { Hajian et al. } \\
(2018)\end{array}$ & QT & $\begin{array}{l}N=150 \\
\text { SA group }(n=50): \text { sui- } \\
\text { cide attempt in the last } \\
12 \text { months } \\
\text { NA group }(n=100) \text { : no } \\
\text { suicide attempt }\end{array}$ & CD-RISC2 & Individual: no suicide attempt. \\
\hline $\begin{array}{l}\text { Herrero et al. } \\
\text { (2018) }\end{array}$ & QT & $\begin{array}{l}N=2376 \\
\text { Resilient group } \\
(n=1624): \text { women who } \\
\text { suffered IPV only with } \\
\text { previous partner } \\
\text { Non-resilient group } \\
(n=594): \text { previous and } \\
\text { current IPV }\end{array}$ & $\begin{array}{l}\text { Survey on women's well-I } \\
\text { being and safety (European } \\
\text { Union Agency for Funda-t } \\
\text { mental Rights[FRA], 2014). }\end{array}$ & $\begin{array}{l}\text { Individual: age, depression*, and anxiety*. } \\
\text { Relational: greater severity of physical violence, violence as } \\
\text { the motivation for ending the relationship, increase in the } \\
\text { length of the current relationship*, child abuse*, victimiza- } \\
\text { tion by non-partners*. } \\
\text { Contextual: country with higher human development (in } \\
\text { terms of health, education, and wealth), dissatisfaction with } \\
\text { income*. }\end{array}$ \\
\hline $\begin{array}{l}\text { Hodges \& } \\
\text { Cabanilla (2011) }\end{array}$ & QT & $N=74$ & RS & Relational: help-seeking. \\
\hline
\end{tabular}




\begin{tabular}{|c|c|c|c|c|}
\hline Article & Design & Participants & Instrument & Factors associated with resilience \\
\hline$\overline{\text { Hou et al. (2016) }}$ & QT & $\begin{array}{l}N=24 \\
\text { Experimental group } \\
(n=8): \text { received } 8 \text { weeks } \\
\text { of a strengths-based } \\
\text { group intervention } \\
\text { Control group }(n=16): \\
\text { no intervention }\end{array}$ & RS & Contextual: strengths-based intervention. \\
\hline $\begin{array}{l}\text { Howell et al. } \\
\text { (2018) }\end{array}$ & QT & $N=112$ & CD-RISC & $\begin{array}{l}\text { Individual: spirituality. } \\
\text { Relational: a greater number of violent relationships*, in- } \\
\text { formal support. } \\
\text { Contextual: education. }\end{array}$ \\
\hline $\begin{array}{l}\text { Humphreys } \\
\text { (2003) }\end{array}$ & QT & $N=50$ & RS & $\begin{array}{l}\text { Individual: perceived physical health, presence and severity } \\
\text { of psychological distress and somatization*, obsessive- } \\
\text { compulsive symptoms*, interpersonal sensitivity*, depres- } \\
\text { sion*, anxiety*. }\end{array}$ \\
\hline $\begin{array}{l}\text { Jaramillo-Vélez et } \\
\text { al. (2005) }\end{array}$ & & $N=199$ & $\begin{array}{l}\text { i } \\
\text { t } \\
\text { t } \\
\text { t } \\
\text { t }\end{array}$ & $\begin{array}{l}\text { Individual: } 20-29 / 50-59 \text { years old, spirituality, psycholog- } \\
\text { ical distress and somatization*, obsessive-compulsive symp- } \\
\text { toms*, interpersonal sensitivity*, depression*, anxiety*, hos- } \\
\text { tility*, phobic anxiety*, paranoid ideation*, psychoticism*. } \\
\text { Relational: no children, married. } \\
\text { Contextual: secondary, technological or university educa- } \\
\text { tion. }\end{array}$ \\
\hline $\begin{array}{l}\text { Jose \& Novaco } \\
(2015)\end{array}$ & QT & $N=136$ & CD-RISC & Individual: perceived stress*, depression*, anxiety*, anger*. \\
\hline $\begin{array}{l}\text { Kramer et al. } \\
(2015)\end{array}$ & QT & $N=181$ & \multicolumn{2}{|l|}{$\begin{array}{l}\text { Davidson Trauma ScaleI } \\
\text { (DTS; Davidson et al., } \\
\text { 1997). } \\
\text { The Center for Epidemio- } \\
\text { logic Studies Depression } \\
\text { Scale (CES- D; Radloff, } \\
\text { 1977). } \\
\text { John Henry Active Coping } \\
\text { Scale(JHAC12; James et al., } \\
\text { 1983). }\end{array}$} \\
\hline $\begin{array}{l}\text { Kuijpers et al. } \\
(2012)\end{array}$ & QT & $N=156$ & \multicolumn{2}{|l|}{$\begin{array}{l}\text { The World Health Organi-I } \\
\text { zation Quality of Life Scale } \\
\text { abbreviated } \\
\text { (WHOQOL- version } \\
\text { WHOQOL Group, 1998). }\end{array}$} \\
\hline $\begin{array}{l}\text { Machisa et al. } \\
\text { (2018) }\end{array}$ & QT & $N=189$ & \multicolumn{2}{|c|}{$\begin{array}{l}\text { The Center for Epidemio-Individual: ability to find money in an emergency, binge } \\
\text { logic Studies Depressiondrinking*. } \\
\text { Scale (CES- D; Radloff,Relational: negative family reactions*, severe IPV in the } \\
\text { 1977). } \\
\text { Harvard Trauma Ques-Contextual: use of medical or psychosocial services*, sup- } \\
\text { tionnaire (Mollica et al.,portive community. } \\
\text { 1992). }\end{array}$} \\
\hline $\begin{array}{l}\text { McFarlane et al. } \\
\text { (2014) }\end{array}$ & QT & $\begin{array}{l}N=300 \\
\text { Shelter women }(n=150) \\
\text { Protection order appli- } \\
\text { cants }(n=150)\end{array}$ & $\begin{array}{l}\text { Safety Behavior Checklist } \\
\text { (McFarlane \& Parker, } \\
\text { 1994). } \\
\text { General Self-Efficacy Scale } \\
\text { (Schwarzer \& Jerusalem, } \\
\text { 1995). Koci Marginality In- } \\
\text { dex (KMI; Koci, 2004). } \\
\text { Norbeck Social Support } \\
\text { (Norbeck et al., 1981, } \\
\text { 1983). }\end{array}$ & Contextual: access to shelter, seeking a protection order. \\
\hline $\begin{array}{l}\text { Rodríguez et al. } \\
\text { (2008) }\end{array}$ & QT & $\begin{array}{l}N=210 \\
\text { Positive to IPV }(n=92) \\
\text { Negative to IPV }(n= \\
118)\end{array}$ & RS & Individual: depression. \\
\hline
\end{tabular}




\begin{tabular}{|c|c|c|c|c|}
\hline Article & Design & Participants & Instrument & Factors associated with resilience \\
\hline $\begin{array}{l}\text { Roditti et al. } \\
(2010)\end{array}$ & QT & $N=72$ & RS & $\begin{array}{l}\text { Individual: mood disturbance*, feeling unworthy*, believ- } \\
\text { ing that "I can be on my own if I have to". }\end{array}$ \\
\hline $\begin{array}{l}\text { Scrafford et al. } \\
(2019)\end{array}$ & QT & $N=76$ & RRCARM & $\begin{array}{l}\text { Individual: depression and PTSD*, early labor and delivery } \\
\text { in pregnant women*. } \\
\text { Relational: IPV exposure*, childhood adversity*. }\end{array}$ \\
\hline $\begin{array}{l}\text { Schultz et al. } \\
(2009)\end{array}$ & QT & $N=77$ & RS & $\begin{array}{l}\text { Individual: depression, anger, fatigue, confusion and vig- } \\
\text { or*, feeling unworthy*, not believing that "I can be on my } \\
\text { own if I have to"*, women foreigners who had been in } \\
\text { USA longer than ten years*, Anglo vs Hispanic women. } \\
\text { Relational: childhood abuse*. } \\
\text { Contextual: less informational support*, unsupportive } \\
\text { formal services*, acculturation (just a hypothesis). }\end{array}$ \\
\hline
\end{tabular}

Note. QL: Qualitative Study; QT: Quantitative Study; SI: Semi-Structured Interview; OI: Open Interview; FG: Focus Group; O: Observation; LH: Life History; CD-RISC:Connor-Davidson Resilience Scale (Connor \& Davidson, 2003); RS: Resilience Scale (Wagnild \& Young, 1993); DRS 15: Dispositional Resilience Scale (Bartone, 2007);CD-RISC2: Abbreviated version of the Connor-Davidson Resilience Scale (Vaishnavi et al., 2007); RRCARM: The Resilience Research Centre Adult Resilience Measure(Liebenberg et al., 2012).

$*$ Negative association between this variable and resilience.

Figure 2

Main contextual, relational, and individual factors associated with resilience in women survivors of IPV.

\begin{tabular}{|c|c|}
\hline \multicolumn{2}{|c|}{$\begin{array}{c}\text { CONTEXTUAL FACTORS } \\
\text { Sense of belonging } \\
\text { Supportive community } \\
\text { Safety environment } \\
\text { Housing } \\
\text { Employment and education } \\
\text { Informational resources } \\
\text { Formal support }\end{array}$} \\
\hline \multicolumn{2}{|c|}{$\begin{array}{c}\text { RELATIONAL FACTORS } \\
\text { Help-seeking } \\
\text { Rebuilding and expanding social network } \\
\text { Having children } \\
\text { Informal support }\end{array}$} \\
\hline \multicolumn{2}{|c|}{ INDIVIDUAL FACTORS } \\
\hline $\begin{array}{l}\text { Problem focused coping } \\
\text { Self-efficacy } \\
\text { Self-esteem } \\
\text { Sense of control } \\
\text { Sense of humor } \\
\text { Optimism/hope } \\
\text { Proactivity } \\
\text { Adaptability }\end{array}$ & $\begin{array}{l}\text { Creativity } \\
\text { Spirituality } \\
\text { Psychological health } \\
\text { Identity reconstruction } \\
\text { Reframing the violent relationship } \\
\text { Goals } \\
\text { Personal growth }\end{array}$ \\
\hline
\end{tabular}

\section{Discussion}

The global aim of the present study was to analyze if the empirical evidence on resilience in women survivors of IPV is useful for guiding future research and policies, shedding light on what supports are needed by the women to restore control over their lives. In this regard, the findings from this systematic review offer helpful insights.

Although the conceptual confusion around the definition and assessment of Resilience was also reflected in IPV research, studies have identified several correlates of the construct in the individual, relational, and contextual dimensions. This result is in line with what has been found in studies in other populations affected by extreme stress, trauma, 
violence, or low socioeconomic conditions, such as immigrants, refugees, indigenous, and LGBTQ populations (Liebenberg et al., 2017).

The appraised articles found moderate to high resilience scores in women survivors of IPV, which could be explained by the high proportion of studies that recruited sample from support services. Participants' resilience may have been reinforced by the process of help- seeking (Crowe et al., 2016) or by the support they received from services (WHO, 2018).

Research must consider this distinction, given that not every woman who suffers from IPV has access to the same quantity and quality of resilience resources.

The individual variables and processes found by this systematic review (e.g., reframing the relationship, identity reconstruction, setting goals, self-efficacy, etc.) are in line with the results from classical research on the process of leaving an abusive relationship (Burke et al., 2004; Enander \& Holmberg, 2008; Landenburger, 1989; Merritt-Gray \& Wuest, 1995; Moss et al., 1997). Sense of control stands out as one of the principal sources of resilience. Since trauma destroys a woman's control over her own life, restoring power to the survivor is a key part of the recovery process (Herman, 1992). To resist and survive violence, women adapt to the circumstances, fluctuating between a powerless/victim identity and a powerful/survivor identity in a nonlinear process (Van Schalkwyk et al., 2014). Sense of control influences the help-seeking process in a complex way: perceived low control is related to the need for help but actual help-seeking behavior seems to be associated with a prior higher sense of control (Katerndahl et al., 2019). Once the violent relationship has ended, levels of coercive control decrease and women's space for action expands, especially with respect to psychological well-being and relationships, but it is important to clarify that through the recovery process women's space for action is sometimes constrained by structural barriers (Sharp-Jeffs et al., 2018).

In the relational and contextual dimensions, help-seeking and support obtained from informal and formal networks are essential components, since they promote access to informational, instrumental, material, and emotional resources, as established in other studies(DGVG, 2019; Lelaurain et al., 2017).

Previous research has shown that one of the main motivators for help-seeking is the presence of children (Fontanil et al., 2020; Katerndahl et al., 2019; Wood et al., 2019). However, other studies have found that having children can be an obstacle in the process of breaking up the violent relationship (DGVG, 2019; Domenech del Río \& Sirvent-García del Valle, 2016; Lelaurain et al., 2017). As recognized by the Council of Europe in Resolution 1714 (2010), every child witnessing violence against their mother is a victim of a form of psychological abuse with potentially severe consequences. The present systematic review shows that women become aware of the effects of violence on their children and seek help for their well-being. Empirical evidence points to an urgency for institutional holistic responses to IPV, and for col- laboration between child welfare and IPV agencies to address women and children's needs (Langenderfer-Magruder et al., 2019; Mennicke et al., 2018).

Finally, employment, education, housing, and a safe environment are facilitators of resilience in women survivors of IPV, while other macrosystemic variables, such as gender roles, social attitudes, and different structural barriers, negatively influence the process of leaving the violence. These results are consistent with previous research in the field, which endorses the adoption of policies to provide women and children with instrumental, material, and informational support and to fight gender discrimination and social attitudes that are favorable to violence (DGVG, 2019; Fontanil et al., 2020; Lelaurain et al., 2017; WHO, 2018). Political actions taken at the macrosystemic level not only enhance the contextual resilience but also reinforce resilience in the individual and relational spheres, since all ecological systems are interrelated (Bronfenbrenner, 1979).

\section{Limitations}

This is the first systemic review to explore factors associated with resilience in women survivors of IPV. It has identified an exhaustive list of existing studies, providing a useful summary of their content. This review, however, is not without its limitations.

The first limitation relates to conceptual and methodological issues in some of the reviewed articles: for example, the use of convenience, nonrepresentative, and small-sized samples; the use of retrospective assessment (risk of recall bias); and in some cases, the use of non-standardized IPV assessment and data collection based exclusively on women's reports with no triangulation (risk of social desirability and/or recall bias).

In addition, the risk of bias in the articles included in this systematic review was not evaluated. Future research should assess the quality and risk of bias in the selected studies, as recommended by Moher et al. (2009) and Ruiz-Aparicio et al. (2018). However, it is important to point out that the researchers who carried out this systematic review carefully appraised the bibliographical records from the databases to ensure a high level of quality.

Further, hand-searching and reference list scanning were carried out to minimize bias and increase the quality of the systematic review (Vassar et al., 2016). It would also have been useful to perform the searches in additional databases to reduce the risk of publication bias. Finally, by selecting empirical evidence published only in English or Spanish, information contained in studies written in other languages was overlooked.

\section{Further research}

Future quantitative studies with large sample sizes should test integrative resilience models in order to obtain a consensual, operative, multidimensional, contextual, and process- 
oriented definition, as recommended by experts in the field (Bushati, 2020; Chmitorz et al., 2018; Stainton et al., 2018; Ungar, 2019; Ungar \& Theron, 2019). Further, with recent research supporting the idea of resilience as a phenomenon that extends beyond the appearance of the stressor event (Chmitorz et al., 2018), longitudinal investigations monitoring resilience in different phases and after the break up are needed. Qualitative studies could be useful in this regard.

Research should assess IPV by using standardized instruments or comprehensive interviews carried out by experts in the field, properly distinguishing coercive control situations from situational couple violence (Johnson, 2008). Studies should also record and consider the type of IPV (psychological/emotional, physical, sexual), the duration of the violence, and the time elapsed since the end of the relationship. Some authors highlight the need to explore the violent behaviors of women to better understand the context of the violence (Herrero et al., 2018; Howell et al., 2018; Kuijpers et al., 2012). In this respect, further research could triangulate the information with data provided by other sources.

Research on resilience needs to delve into the mechanisms through which vulnerability or protective factors exert their influence on groups with specific risk conditions. The particularities of the adversity and how these interact with the personal and environmental characteristics of the people concerned make it difficult to extrapolate findings from one sample to another. Therefore, it is important to consider the heterogeneity of adverse events that may be experienced by women. Future research should adopt an ecological perspective and analyze macrosystemic factors and policies, considering the accessibility and availability of resources at each ecological level (Bushati, 2020; Fontanil et al., 2020; Ungar, 2019; Ungar \& Theron, 2019). It would also be interesting to incorporate an ethnographic focus to explore the relationship between race or ethnicity and resilience.

Finally, the feminist perspective could enrich studies by drawing attention to women's voices. Consulting women and working with their narratives and ideas is an appropriate way to build guidelines for professional interventions and policy practices (Crawford et al., 2009; Davis, 2002; Fontanil et al., 2020; Leung et al., 2019).

In summary, it has been detected that research on this field have had a stronger focus on individual resilience factors when compared with the study of contextual variables, therefore it becomes necessary to deeply explore resilience from a contextual perspective that includes ecological mesosystemic, exosystemic, and macrosystemic factors. Fur-

\section{References}

*Ahmad, F., Rai, N., Petrovic, B., Erickson, P. E., \& Stewart, D. E. (2013). Resilience and resources among South Asian immigrant women as survivors of partner violence. Journal of Immigrant and Minority Health, 15(6), 1057-1064. https://doi.org/10.1007/s10903-013-9836-2

*Alvi, S., Clow, K. A., \& DeKeseredy, W. S. (2008). Women abuse and resilience in a sample of minority low-income women. Women's Health \& thermore, it is essential to analyze the influence of multiple heterogeneous women's vulnerability conditions that could potentially affect resilience, such as race, ethnicity, and disability. Finally, there is also a lack of long-term studies following women after the end of the relationship. It could be beneficial to full this gap by analyzing women's resilience in the post-separation phase, especially when they must keep in contact with their former partner because of the presence of children in common.

\section{Conclusions}

The study contributes significantly to the body of literature by synthesizing existing empirical evidence on the factors of resilience in women survivors of IPV. To our knowledge, this is the first systematic review on this topic with this specific population. Drawn on the reviewed articles, this study provides support for multidimensional, contextual, and process-oriented explanations of resilience in women survivors of IPV. Individual, but also relational and contextual factors are core in women's resilience and should be considered for guiding future research and policies.

Given the gravity of IPV, recognized as a serious public health issue affecting a high proportion of women around the world, governments and institutions should take actions to support women and children and to prevent future IPV. Evidence-based policies and professional practices are necessary to facilitate women's access to education, employment, and housing, which would promote their independence and sense of control, as well as protect them from revictimization. If violence undermines women and children's psychological and physical well-being, then society should ensure survivors have access to professional health care. It is also important to design prevention programs and implement them on an ongoing basis and at all ecological levels, since violence is a multicausal and multidimensional phenomenon.

Acknowledgments.- This work was supported by the Spanish Ministry of Science, Innovation, and Universities (FPU scholarship19/00441), The State Bureau of Investigations, and the European Regional development fund (RTI2018-096476-B-100).

The authors would like to thank Joel Juarros-Basterretxea (Facultad de Educación de la Universidad de Zaragoza) for the valuable comments and suggestions.

Conflict of interest.- The authors declare no conflict of interest.

Urban Life, $\quad 7(2), \quad 51-67$. https://tspace.library.utoronto.ca/bitstream/1807/16689/1/Alvi_Clo w_DeKeseredy.pdf

*Anderson, K. M., Renner, L. M., \& Danis, F. S. (2012). Recovery: Resilience and growth in the aftermath of domestic violence. Violence Against 
Women

18(11),

https://doi.org/10.1177/1077801212470543

Arias, E., Velasco, J., \& Novo, M. (2016). Análisis bibliométrico sobre la investigación en violencia de género. Fundamentos y nuevas tendencias Bibliometric analysis on gender violence. Fundamentals and new trends]. In A. Andrés-Pueyo, F. Farina, M. Novo, \& D. Seijo (Eds.), Avances en psicologia jurídica y forense. Colección Psicología y Ley [Advances in legal and forensic psychology. Psychology and law collection] (pp. 81-91). Sociedad Española de Psicología Jurídica y Forense. https://doi.org/10.13140/RG.2.1.2594.2807

Fontanil, Y., \& Alcedo, M. A. (2018). Protocolo contra la violencia sexual del Principado de Asturias [Protocol against sexual violence of the Principality of Asturias]. Gobierno del Principado de Asturias, Instituto Asturiano de la Mujer. https://iam.asturias.es/documents/269132/273392/PROTOCOLOCONTRA-LA- VIOLENCIA-SEXUAL-PRINCIPADO-DEASTURIAS-2018.pdf/6cd8e4e5-ffbb-589b- 7b6f-437d71331300

Fontanil, Y., Alcedo, M. A., Fernández-Álvarez, N., Ezama, E., \& Fernández, R. (2020). Salidas seguras para mujeres víctimas de violencia de género en la pareja [Safe exits for women victims of gender violence in the couple]. KrK Ediciones.

Bartone, P. T. (2007). Test-retest reliability of the Dispositional Resilience Scale-15, a brief hardiness scale. Psychological Reports, 101(3), 943-944. https://doi.org/10.2466/pr0.101.3.943-944

Bonanno, G. A. (2004). Loss, trauma, and human resilience: Have we underestimated the human capacity to thrive after extremely aversive events? American Psychologist, 59(1), 20-28. https://doi.org/10.1037/0003066X.59.1.20

Bronfenbrenner, U. (1979). The ecology of human development. Harvard University Press.

Burke, J. G., Denison, J. A., Gielen, A. C., McDonnell, K. A., \& O'Campo, P. (2004). Ending intimate partner violence: An application of the transtheoretical model. American Journal of Health Behavior, 28(2), 122 133. https://doi.org/10.5993/AJHB.28.2.3

Bushati, A. (2020). Exploring the labyrinths of resilience: A concept analysis. European Journal of Social Science, Education and Research, 7(2), 113-126. http://journals.euser.org/files/articles/ejser_v7_i2_20/Bushati.pdf

*Canaval, G. E., González, M. C., \& Sánchez, M. O. (2007). Espiritualidad y resiliencia en mujeres maltratadas que denuncian su situación de violencia de pareja [Spirituality and Resilience in battered women that denounce intimate partner violence]. Colombia Medica, 38(4)72-78. http://www.scielo.org.co/scielo.php?script=sci_arttext\&pid=S165795342007000600008

*Canaval, G. E., González, M. C., Humphreys, J., De León, N., \& González, S. (2009). Violencia de pareja y salud de las mujeres que consultan a las comisarías de familia, Cali, Colombia [Partner violence and women's health reported to family commissaries, Cali, Colombia]. Investigación y Educación en Enfermería, 27(2), 209-217. http://www.scielo.org.co/scielo.php?script=sci_arttext\&pid=S012053072009000200006

*Cesario, S. K., Nava, A., Bianchi, A., McFarlane, J., \& Maddoux, J. (2014). Functioning outcomes for abused immigrant women and their children 4 months after initiating intervention. Revista Panamericana de Salud Pública-Panamerican Journal of Public Health, 35(1), 8-14. https://www.researchgate.net/publication/260809531_Functioning_o utcomes_of_abused_immigrant_. en_and_their_children_4_months_after_initiating_intervention

Chmitorz, A., Kunzler, A., Helmreich, I., Tüscher, O, Kalisch, R., Kubiak, T., Wessa, M., \& Lieb, K. (2018). Intervention studies to foster resilience-A systematic review and proposal for a resilience framework in future intervention studies. Clinical Psychology Review, 59, 78-100. https://doi.org/10.1016/j.cpr.2017.11.002

*Choi, A. W. M., Liu, L. L., Chan, P. Y., Lo, R. T. F., Wong, J. Y. H., \& Tang, D. H. M. (2018). The Significance of volunteer support for female survivors recovering from intimate-partner violence. British Journal of Social Work, 49(7), 1778-1797. https://doi.org/10.1093/bjsw/bcy109

*Choi, A. W. M., Lo, B. C. Y., Lo, R. T. F., To, P. Y. L., \& Yuen-Ha Wong, J. (2019). Intimate partner violence victimization, social support, and resilience: Effects on the anxiety levels of young mothers. Journal of Interpersonal Violence, 886260519888532. Advance online publication. https://doi.org/10.1177/0886260519888532
Connor, K. M., \& Davidson, J. R. T. (2003). Development of a new resilience scale: The Connor-Davidson Resilience Scale (CD-RISC). Depression and Anxiety, 18(2), 76-82. https://doi.org/10.1002/da.10113

Council of Europe. (2010). Resolution 1714: Children who witness domestic violence. Council ofEurope. https://assembly.coe.int/nw/xml/XRef/XrefXML2HTML- en.asp?fileid=17826\&lang=en

*Crann, S. E., \& Barata, P. C. (2016). The experience of resilience for adul female survivors of intimate partner violence: A phenomenological inquiry. Violence Against Women, 22(7), 853- 875. https://doi.org/10.1177/1077801215612598

*Crawford, E., Liebling-Kalifani, H., \& Hill, V. (2009). Women's understanding of the effects of domestic abuse: The impact on their identity, sense of self and resilience. A grounded theory approach. Journal of Inter national Women's $\quad$ Studies, 11(2), 63-82. https: / vc.bridgew.edu/cgi/viewcontent.cgi?article=1171\&context=jiw

Crowe, A., Averett, P., \& Glass, J. S. (2016). Mental illness stigma, psychological resilience, and help seeking: What are the relationships? Mental Health o Prevention, 4(2), 63-68. https://doi.org/10.1016/j.mhp.2015.12.001

Davidson, J. R., Book, S. W., Colket, J. T., Tupler, L. A., Roth, S., David, D., Hertzberg, M., Mellman, T., Beckham, J. C., Smith, R. D., Davison, R. M., Katz, R., \& Feldman, M. E.(1997). Assessment of a new self-rating scale for post-traumatic stress disorder. Psychological Medicine, 27(1), 153160. https://doi.org/10.1017/s0033291796004229

*Davis, R. (2002). "The strongest women": Exploration of the inner resources of abused women. Qualitative Health Research, 12(9), 1248-1263. https://doi.org/10.1177/1049732302238248

*de la Rosa, I. A., Barnett-Queen, T., Messick, M., \& Gurrola, M. (2015). Spirituality and Resilience among Mexican American IPV survivors. Journal of Interpersonal Violence, 31(20), 3332- 3351. https://doi.org/10.1177/0886260515584351

Delegación del Gobierno contra la Violencia de Género. (2019). Estudio sobre el tiempo que tardan las mujeres víctimas de violencia de género en verbalizar su situacion S Study on the time it takes for women victims of gender violence to verbalize their situation]. Centro de Publicaciones del Ministerio de la Presidencia, Relaciones con las Cortes e Igualdad. https://violenciagenero.igualdad.gob.es/violenciaEnCifras/estudios/inv estigaciones/2019/pdfs/Estudio_Tiempo_Denuncia4.pdf

Domenech del Río, I., \& Sirvent-García del Valle, E. (2016). Influence of intimate partner violence severity on the help-seeking strategies of female victims and the influence of social reactions to violence disclosure on the process of leaving a violent relationship. Journal of Interpersonal Violence, 34(21-22), 4550-4571. https://doi.org/10.1177/0886260516676473

*Drumm, R., Popescu, M., Cooper, L., Trecartin, S., Seifert, M., Foster, T. \& Kilcher, C. (2014)."God just brought me through it": Spiritual coping strategies for resilience among intimate partner violence survivors. Clinical Social Work Journal, 42(4), 385-394. https://doi.org/10.1007/s10615-013-0449-y

Enander, V., \& Holmberg, C. (2008). Why does she leave? The leaving process(es) of battered women. Health Care for Women International, 29(3), 200-226. https://doi.org/10.1080/07399330801913802

European Union Agency for Fundamental Rights. (2014). Violence against Women: An EU-wide survey. Survey methodology, sample, and fieldwork. Publications Office of the European Union. https:/ / fra.europa.eu/sites/default/files/fra-2014-vaw-surveytechnical-report-1_en.pdf

*Fernández-Sánchez, M., \& López-Zafra, E. (2019). The voices that should be heard: A qualitative and content analysis to explore resilience and psychological health in victims of intimate partner violence against women (IPVAW). Women's Studies International Forum, 72, 80-86. https://doi.org/10.1016/j.wsif.2018.12.005

*Fogarty, A., Woolhouse, H., Giallo, R., Wood, C., Kaufman, J., \& Brown, S. (2019). Mothers' experiences of parenting within the context of intimate partner violence: Unique challenges and resilience. Journal of Inter personal Violence, 886260519883863. Advance online publication. https://doi.org/10.1177/0886260519883863

*Ford-Gilboe, M., Wuest, J., Varcoe, C., Davies, L., Merritt-Gray, M., Campbell, J., \& Wilk, P.(2009). Modelling the effects of intimate part- 
ner violence and access to resources on women's health in the early years after leaving an abusive partner. Social Science \& Medicine, 68(6), 1021-1029. https://doi.org/10.1016/j.socscimed.2009.01.003

Gondolf, E. W., \& Fisher, E. R. (1988). Battered women as survivors: An alternative to treating learned helplessness. Lexington Books.

*Gopal, N., \& Nunlall, R. (2017). Interrogating the resilience of women affected by violence. Agenda: Empowering Women for Gender Equity, 31(2), 63-73. https://doi.org/10.1080/10130950.2017.1379759

*Hajian, S., Kasaeinia, S., \& Doulabi, M. A. (2018). The effect of resilience and stress coping styles on suicide attempts in females reporting spouse-related abuse. Iranian Journal of Psychiatry and Behavioral Sciences, 12(3), Article e13091. https://doi.org/10.5812/ijpbs.13091

Herman, J. (1992). Trauma and recovery. The aftermath of violence-From domestic abuse to political terror. Basic Books.

*Herrero, J., Vivas, P., Torres, A., \& Rodríguez, F. J. (2018). When violence can appear with different male partners: Identification of resilient and non-resilient women in the European Union. Frontiers in Psychology, 9, 111. https://doi.org/10.3389/fpsyg.2018.00877

*Hodges, T. A., \& Cabanilla, A. S. (2011). Factors that impact help-seeking among battered Blackwomen: Application of critical and survivor theories. Journal of Cultural Diversity, 18(4), 120-125.

*Hou, W.-L., Ko, N.-Y., \& Shu, B.-C. (2016). Effects of a strengths-based perspective support group among Taiwanese women who left a violent intimate partner relationship. Journal of Clinical Nursing, 25(3-4), 543-554. https://doi.org/10.1111/jocn.13091

*Howell, K. H., Thurston, I. B., Schwartz, L. E., Jamison, L. E., \& Hasselle, A. J. (2018). Protective factors associated with resilience in women exposed to intimate partner violence. Psychology of Violence, 8(4), 438-447. https://doi.org/10.1037/vio0000147

Hu, T., Zhang, D., \& Wang, J. (2015). A meta-analysis of the trait resilience and mental health. Personality and Individual Differences, 76, 18-27. https://doi.org/10.1016/j.paid.2014.11.039

*Humphreys, J. (2003). Resilience in sheltered battered women. Issues in Mental Health Nursing, 24(2), 137-152. https://doi.org/10.1080/01612840305293

James, S. A., Hartnett, S. A., \& Kalsbeek, W. D. (1983). John Henryism and blood pressure differences among black men. Journal of Behavioral Medicine, 6(3), 259-278. https://doi.org/10.1007/BF01315113

*Jaramillo-Vélez, D. E., Ospina-Muñoz, D. E., Cabarcas-Iglesias, G., \& Humphreys, J. (2005). Resiliencia, espiritualidad, aflicción y tácticas de resolución de conflictos en mujeres maltratadas [Resilience, spirituality, distress and tactics for battered women's conflict resolution]. Revista de Salud Pública, 7(3), 281-292. http://www.scielo.org.co/scielo.php?script=sci_arttext\&pid=S012400642005000300004

Johnson, M. P. (2008). A typology of domestic violence: Intimate terrorism, violent resistance, and situational couple violence. Northeastern University Press.

*Jose, R., \& Novaco, R. W. (2015). Intimate partner violence victims seeking a temporary restraining order: Social support and resilience attenuating psychological distress. Journal of Interpersonal Violence, 31(20), 33523376. https://doi.org/10.1177/0886260515584352

Katerndahl, D. A., Burge, S. K., Ferrer, R. L., Becho, J., \& Wood, R. (2019). Predictors of perceived need for actual action taking among women in violent relationships. Journal of Interpersonal Violence, 34(16), 3344-3371. https://doi.org/10.1177/0886260516669543

Koci, A. (2004). Marginality, abuse and adverse bealth outcomes in women (Publication No. 3123338) [Doctoral Dissertation, Emory University]. ProQuest Dissertations and Theses Global. Marginality, abuse and adverse health outcomes in women - ProQuest

*Kramer, N. M., Johnson, N. L., \& Johnson, D. M. (2015). Is John Henryism a resilience factor in women experiencing intimate partner violence? Anxiety, Stress and Coping, 28(6), 601-616. https://doi.org/10.1080/10615806.2014.1000879

*Kuijpers, K. F., Van der Knaap, L. M., \& Winkel, F. W. (2012). Victims' influence on intimate partner violence revictimization: An empirical test of dynamic victim-related risk factors. Journal of Interpersonal Violence, 27(9), 1716-1742. https://doi.org/10.1177/0886260511430389

*Labronici, L. M. (2012). Resilience in women victims of domestic violence: A phenomenological view. Texto e Contexto Enfermagem, 21(3), 625-632. https://doi.org/10.1590/S0104- 07072012000300018
Landenburger, K. (1989). A process of entrapment in and recovery from an abusive relationship. Issues in Mental Health Nursing, 10(3), 209-227. https://doi.org/10.3109/01612848909140846

Langenderfer-Magruder, L., Alven, L., Wilke, D. J., \& Spinelli, C. (2019). Getting everyone on the same page: Child welfare workers'collaboration challenges on cases involving intimate partner violence. Journal of Family Violence, 34, 21-31. https://doi.org/10.1007/s10896-018-0002-4

Lelaurain, S., Graziani, P., \& Lo Monaco, G. (2017). Intimate partner violence and help-seeking. A systematic review and social psychological tracks for future research. European Psychologist, 22(4), 263-281. https://doi.org/10.1027/1016-9040/a000304

Leung, L., Miedema, S., Warner, X., Homan, S., \& Fulu, E. (2019) Making feminism count: Integrating feminist research principles in large-scale quantitative research on violence against women and girls. Gender $\& D e-$ velopment, 27(3), 427-447. https://doi.org/10.1080/13552074.2019.1668142

*Lévesque, S., \& Chamberland, C. (2016). Resilience, violence, and early pregnancy: A qualitative study of the processes used by young mothers to overcome adversities. SAGE Open, 6(1), 1- 15. https://doi.org/10.1177/2158244016640851

Liberati, A., Altman, D. G., Tetzlaff, J., Mulrow, C., Gøtzsche, P. C., Ioannidis, J. P. A., Clarke, M., Devereaux, P. J., Kleijnen, J., \& Moher, D. (2009). The PRISMA statement for reporting systematic reviews and metaanalyses of studies that evaluate health care interventions: Explanation and elaboration. PLoS Medicine, 6(7), Article e1000100. https://doi.org/10.1371/journal.pmed.1000100

Liebenberg, L., Joubert, N., \& Foucault, M. L. (2017). Understanding core resilience elements and indicators: A comprehensive review of the literature. Public Health Agency of Canada. http://lindaliebenberg.com/wpcontent/uploads/2017/11/PHAC-Resilience-Report-Final- VersionNovember-2017.pdf

Liebenberg, L., Ungar, M., \& Van de Vijver, F. R. R. (2012). Validation of the Child and Youth Resilience Measure-28 (CYRM-28) among Canadian youth. Research on Social Work Practice, 22(2), 219-226. https://doi.org/10.1177/1049731511428619

*López-Fuentes, I., \& Calvete, E. (2015). Building resilience: A qualitative study of Spanish women who have suffered intimate partner violence. American Journal of Orthopsychiatry, 85(4), 339-351. https://doi.org/10.1037/ort0000070

*Machisa, M. T., Christofides, N., \& Jewkes, R. (2018). Social support factors associated with psychological resilience among women survivors of intimate partner violence in Gauteng, South Africa. Global Health Action, 11(3) Article e1491114. https://doi.org/10.1080/16549716.2018.1491114

McFarlane, J., \& Parker, B. (1994). Abuse during pregnancy: A protocol for prevention and intervention. March of Dimes.

*McFarlane, J., Symes, L., Maddoux, J., Gilroy, H., \& Koci, A. (2014). Is length of shelter stay and receipt of a protection order associated with less violence and better functioning for abused women? Outcome data 4 months after receiving services. Journal of Interpersonal Violence, 29(15), 2748-2774. https://doi.org/10.1177/0886260514526060

Mennicke, A., Langenderfer-Magruder, L., \& MacConnie, L. (2018). "It's tricky...": Intimate partner violence service providers' perspectives of assessments and referrals by child welfare workers. Journal of Family Violence, 34(1), 47-54. https://doi.org/10.1007/s10896-018-9991- 2

Merritt-Gray, M., \& Wuest, J. (1995). Counteracting abuse and breaking free: The process of leaving revealed through women's voices. Health Care for Women International, 16(5), 399-412. https://doi.org/10.1080/07399339509516194

Moher, D., Liberati, A., Tetzlaff, J., Altman, D. G., \& The PRISMA Group (2009). Preferred Reporting Items for Systematic Reviews and MetaAnalyses: The PRISMA statement. Annals of Internal Medicine, 151, 264 269. https://doi.org/10.1371/journal.pmed.1000097

Mollica, R. F., Caspi-Yavin, Y., Bollini, P., Truong, T., Tor, S., \& Lavelle, J. (1992). The Harvard Trauma Questionnaire. Validating a cross-cultural instrument for measuring torture, trauma, and posttraumatic stress disorder in Indochinese refugees. The Journal of Nervous and Mental Disease, 180(2), 111-116. https://doi.org/10.1097/00005053-199202000-00008 
Moss, V. A., Pitula, C. R., Campbell, J. C., \& Halstead, L. (1997). The experience of terminating an abusive relationship from an Anglo and African American perspective: A qualitative descriptive study. Issues in Mental Health Nursing, 18(5), 433-454. https://doi.org/10.3109/01612849709009423

Norbeck, J. S., Lindsey, A. M., \& Carrieri, V. L. (1981). The development of an instrument to measure social support. Nursing Research, 30(5), 264 269. https://doi.org/10.1097/00006199-198109000-00003

Norbeck, J. S., Lindsey, A. M., \& Carrieri, V. L. (1983). Further development of the Norbeck Social Support Questionnaires: Normative data and validity testing. Nursing Research, 32(1), 4-9. https://doi.org/10.1097/00006199-198301000-00002

Radloff, L. S. (1977). The CES-D Scale: A self-report depression scale for research in the general population. Applied Psychological Measurement, 1(3), 385-401. https://doi.org/10.1177/014662167700100306

Raphael, J., \& Tolman, R. (1997). Trapped by poverty/trapped by abuse: New evidence documenting the relationship between domestic violence and welfare. Taylor Institute.

*Roditti, M., Schultz, P., Gillette, M., \& de la Rosa, I. (2010). Resiliency and social support networks in a population of Mexican American intimate partner violence survivors. Families in Society: The Journal of Contemporary Social Services, 91(3), 248-256. https://doi.org/10.1606/1044-3894.4002

*Rodríguez, M. A., Heilemann, M. V., Fielder, E., Ang, A., Nevarez, F., \& Mangione, C. M. (2008). Intimate partner violence, depression, and PTSD among pregnant Latina women. Annals of Family Medicine, 6(1), 44-52. https://doi.org/10.1370/afm.743

Rubio-Aparicio, M., Sánchez-Meca, J., Marín-Martínez, F., \& López-López, J. A. (2018). Recomendaciones para el reporte de revisiones sistemáticas y meta-análisis. [Guidelines for reporting systematic reviews and metaanalyses]. Anales de Psicología, 34(2), 412-420. https://doi.org/10.6018/analesps.34.2.320131

*Schaefer, L. M., Howell, K. H., Sheddan, H. C., Napier, T. R., Shoemaker, H. L., \& Miller-Graff, L. E. (2019). The road to resilience: Strength and coping among pregnant women exposed to intimate partner violence. Journal of Interpersonal Violence. https://doi.org/10.1177/0886260519850538

*Schultz, P., Roditti, M., \& Gillette, M. (2009). Resilience, social support, and psychological disturbance in Hispanic women residing in a battered women's shelter on the U.S./Mexicoborder. Hispanic Health Care International, 7(4), 224-230. https://doi.org/10.1891/1540- 4153.7.4.224

Schwarzer, R., \& Jerusalem, M. (1995). Generalized Self-Efficacy Scale. In J. Weinman, S. Wright, \& M. Johnston (Eds.), Measures in bealth psychology: A user's portfolio (pp. 35-37). NFER-NELSON.

*Scrafford, K. E., Grein, K., \& Miller-Graff, L. E. (2019). Effects of intimate partner violence, mental health, and relational resilience on perinatal health. Journal of Traumatic Stress, 32(4), 506-515. https://doi.org/10.1002/jts.22414

* Shanthakumari, R. S., Chandra, P. S., Riazantseva, E., \& Stewart, D. E (2014). "Difficulties come to humans and not trees, and they need to be faced": A study on resilience among Indian women experiencing intimate partner violence. International Journal of Social Psychiatry, 60(7), 703710. https://doi.org/10.1177/0020764013513440

Sharp-Jeffs, N., Kelly, L., \& Klein, R. (2018). Long journeys toward freedom: The relationship between coercive control and space for actionmeasurement and emerging evidence. Violence Against Women, 24(2), 163-185. https://doi.org/10.1177/1077801216686199

Stainton, A., Chisholm, K., Kaiser, N., Rosen, M., Upthegrove, R., Ruhrmann, S., \& Wood, S. J. (2019). Resilience as a multimodal dynamic process. Early Intervention in Psychiatry, 13(4), 725-732. https://doi.org/10.1111/eip.12726

*Taylor, J. Y. (2004). Moving from surviving to thriving African American women recovering from intimate male partner abuse. Research and Theory for Nursing Practice: An International Journal, 18(1), 35-50. https://doi.org/10.1891/rtnp.18.1.35.28056

*Trigueiro, T. H., Labronici, L. M., Merighi, M. A. B., \& Raimondo, M. L. (2014). The process of resilience in women who are victims of domestic violence: A qualitative approach. CogitareEnfermagem, 19(3), 395-401. https://doi.org/10.5380/ce.v19i3.34726

Ungar, M. (2008). Resilience across cultures. The British Journal of Social Work, 38(2), 218-235. https://doi.org/10.1093/bjsw/bcl343

Ungar, M. (2013). Resilience, Trauma, Context, and Culture. Trauma, Violence, \& Abuse, 14(3), 255-266. https://doi.org/10.1177/1524838013487805

Ungar, M. (2019). Designing resilience research: Using multiple methods to investigate risk exposure, promotive and protective processes, and contextually relevant outcomes for children and youth. Child Abuse \& $\mathrm{Ne}$ glect, $96, \quad$ Article e104098 https://doi.org/10.1016/j.chiabu.2019.104098

Ungar, M., \& Theron, L. (2019). Resilience and mental health: How multisystemic processes contribute to positive outcomes. The Lancet Psychiatry, 7(5), 441-448. https://doi.org/10.1016/S2215-0366(19)30434-1

Vaishnavi, S., Connor, K., \& Davidson, J. R. T. (2007). An abbreviated version of the Connor-Davidson Resilience Scale (CD-RISC), the CD RISC2: Psychometric properties and applications in psychopharmacological trials. Psychiatry Research, 152(2-3), 293-297. https://doi.org/10.1016/j.psychres.2007.01.006

Van Schalkwyk, S., Boonzaier, F., \& Gobodo-Madikizela, P. (2014). "Selves" in contradiction: Power and powerlessness in South African shelter residents' narratives of leaving abusive heterosexual relationships. Feminism \& Psychology, 24(3), 314-331. https://doi.org/10.1177/0959353513514245

Vassar, M., Atakpo, P., \& Kash, M. J. (2016). Manual search approaches used by systematic reviews in dermatology. Journal of Medical Library Association, 104, 302-304. https://doi.org/10.5195/jmla.2016.145

Wagnild, G. M., \& Young, H. M. (1993). Development and psychometric evaluation of Resilience scale. Journal of Nursing Measurement, 1(2), 165 $178 . \quad$ https://sapibg.org/download/1054- wagnild_1993_resilience_scale_2.pdf

*Werner-Wilson, R. J., Zimmerman, T. S., \& Whalen, D. (2000). Resilient response to battering. Contemporary Family Therapy, 22(2), 161-188. https://doi.org/10.1023/A:1007777702757

Wood, S. N., Glass, N., \& Decker, M. R. (2019). An integrative review of safety strategies for women experiencing intimate partner violence in low- and middle-income countries. Trauma, Violence, \& Abuse, 22(1), 6882. https://doi.org/10.1177/1524838018823270

World Health Organization Quality of Life Group. (1998). Development of the World Health Organization WHOQOL-BREF quality of life assessment. Psychological Medicine, 28(3), 551-558. https://doi.org/10.1017/S0033291798006667

World Health Organization. (2013). Global and regional estimates of violence against women: Prevalence and health effects of intimate partner violence and nonpartner sexual violence. World Health Organization. https://apps.who.int/iris/bitstream/handle/10665/85239/978924156 4625_eng.pdf?sequence $=1$

World Health Organization. (2018). Health 2020 priority area four: Creating supportive environments and resilient communities. A compendium of inspirational examples. WHO Regional Office for Europe. https://www.mscbs.gob.es/profesionales/saludPublica/prevPromocion /promocion/desigualda dSalud/docs/resilience-sc-eng.pdf

*Zalapa, E., García, Y., \& Figueroa, G. (2012). Resiliencia en mujeres morelianas separadas por violencia conyugal [Resilience in Morelian women separeted for marital violence]. Revista de Psicología (Trujillo), 14(2), 164 177.

https://sisbib.unmsm.edu.pe/BVRevistas/rev_psicologia_cv/v14_2012 _2/pdf/a03v14n2.pdf 